\title{
LCM from FCA Point of View: A CbO-style Algorithm with Speed-up Features
}

\author{
Radek Janostik, Jan Konecny, Petr Krajča \\ Dept. Computer Science, Palacký University Olomouc \\ 17. listopadu 12, CZ-77146 Olomouc \\ Czech Republic
}

\begin{abstract}
LCM is an algorithm for enumeration of frequent closed itemsets in transaction databases. It is well known that when we ignore the required frequency, the closed itemsets are exactly intents of formal concepts in Formal Concept Analysis (FCA). We describe LCM in terms of FCA and show that LCM is basically the Close-by-One algorithm with multiple speed-up features for processing sparse data. We analyze the speed-up features and compare them with those of similar FCA algorithms, like FCbO and algorithms from the In-Close family.
\end{abstract}

Keywords: algorithm; formal concept analysis; frequent closed itemset; Close-by-One

\section{Introduction}

Frequent closed itemsets in transaction databases are exactly intents in Formal Concept Analysis (FCA) with sufficient support - cardinality of the corresponding extents. If the minimum required support is zero (i.e. any attribute set is considered frequent), one can easily unify these two notions. LCM (Linear time Closed itemset Miner) is an algorithm for the enumeration of frequent closed itemsets developed by Takeaki Uno [17, 18, 19, 20] in 20032005. It is considered to be one of the most efficient algorithms for this task. ${ }^{1}$

We have thoroughly studied Uno's papers and source codes and, in the present paper, we deliver a complete description of LCM from the point of

\footnotetext{
*Corresponding author

Email addresses: radek.janostik@upol.cz (Radek Janostik), jan.konecny@upol.cz (Jan Konecny), petr.krajca@upol.cz (Petr Krajča)

${ }^{1}$ Its implementations with source codes are available at URL http://research.nii. ac.jp/ uno/codes.htm
} 
view of FCA. Despite the source codes being among the main sources for this study, we stay at a very comprehensible level in our description and avoid delving into implementation details. We explain that the basis of LCM is Kuznetsov's Close-by-One (CbO) [13].2 We describe its additional speed-up features and compare them with those of state-of-art CbO-based algorithms, like FCbO [16] and In-Close2+ [4, [5, 6, 7] $:^{3}$

Remark 1 (Some subjective notes on our motivations). Besides the obvious importance of LCM for $\mathrm{FCA}_{4}^{4}$, there are two motivational points for this work. We separated them into this remark as they are based on our subjective impressions and views. We ask the reader to excuse the rather subjective tone in this remark.

(a) A significant part of the FCA community is aware of LCM and uses Uno's implementation to enumerate formal concepts for further processing or for comparison with their methods. However, it is our impression that, more often than not, the implementation is used merely as a black box. We believe that this part of the FCA community would appreciate 'unwrapping the black box'.

(b) Uno's papers provide quite confusing descriptions of LCM and the source codes of the implementations are not written to allow easy analysis. Moreover, Uno's implementation and description of LCM have some differences; there is even an important feature present in the implementation which is not described in the papers. We believe that a clearer and more complete description would be fruitful.

The paper is structured as follows. First, we recall the basic notions and notations of FCA (Section 2) we use in the rest of this paper. Then we describe $\mathrm{CbO}$ (Section 3) as it is a basis for description of LCM. Afterwards, we provide a description of LCM's features (Section 4), namely, initial preprocessing of data (Section 4.1), handling data using arraylists computing all attribute extents at once (Section 4.2), conditional datasets (Section 4.3), and pruning (Section 4.4). In all of the above, we ignore the requirement of

\footnotetext{
${ }^{2}$ Although LCM was most likely developed independently.

${ }^{3}$ In the rest of this paper, whenever we write 'CbO-based algorithms' we mean $\mathrm{CbO}$, $\mathrm{FCbO}$ and In-Close family of algorithms. By version number $2+$, we mean the version 2 and higher.

${ }^{4}$ However, the closed sets are also relevant in other disciplines, like association rule mining [1, 8, condensed representation of inductive queries [15], or logical analysis of data [2, 3.
} 


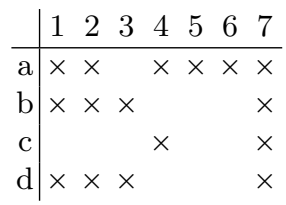

Figure 1: Formal context with objects a,b,c,d and attributes $1,2, \ldots, 7$

frequency and we describe it separately (Section 5). We provide a tutoriallike description of complete FP-trees used for the denser parts of data in LCM3 (Section 6). Finally, we summarize our conclusions (Section 7).

This paper is an extended version of the conference paper [10] presented at CLA 2020.

\section{Formal Concept Analysis}

An input to FCA is a triplet $\langle X, Y, I\rangle$, called a formal context, where $X$ and $Y$ are non-empty sets of objects and attributes, respectively, and $I$ is a binary relation between $X$ and $Y ;\langle x, y\rangle \in I$ means that the object $x$ has the attribute $y$. Finite formal contexts are usually depicted as tables, in which rows represent objects, columns represent attributes, and each entry contains a cross if the corresponding object has the corresponding attribute, and is left blank otherwise; see Fig.1 for an example. In this paper, we consider only finite formal contexts.

The formal context induces concept-forming operators:

$\uparrow: \mathbf{2}^{X} \rightarrow \mathbf{2}^{Y}$ assigns to a set $A$ of objects the set $A^{\uparrow}$ of all attributes shared by all the objects in $A$.

$\downarrow: \mathbf{2}^{Y} \rightarrow \mathbf{2}^{X}$ assigns to a set $B$ of attributes the set $B^{\downarrow}$ of all objects which share all the attributes in $B$.

Formally,

$A^{\uparrow}=\{y \in Y \mid \forall x \in A:\langle x, y\rangle \in I\}, \quad$ and $\quad B^{\downarrow}=\{x \in X \mid \forall y \in B:\langle x, y\rangle \in I\}$.

For singletons, we use shortened notation and write $x^{\uparrow}, y^{\downarrow}$ instead of $\{x\}^{\uparrow}$, $\{y\}^{\downarrow}$, respectively.

In this paper, we assume a set of attributes $Y=\{1, \ldots, n\}$. Whenever we write about lower and higher attributes, we refer to the natural ordering $\leqslant$ of the numbers in $Y$. 
A formal concept is a pair $\langle A, B\rangle$ of sets $A \subseteq X, B \subseteq Y$, such that $A^{\uparrow}=B$ and $B^{\downarrow}=A$. The first component of a formal concept is called the extent, whilst the second one is called the intent.

The compositions ${ }^{\uparrow}$ and $\downarrow \uparrow$ of concept-forming operators are closure operators on $2^{X}$ and $2^{Y}$, respectively. That is, the composition ${ }^{\downarrow \uparrow}$ satisfies

$$
\begin{array}{rc}
\text { (extensivity) } & \subseteq \subseteq B^{\downarrow \uparrow} \\
\text { (monotony) } & B \subseteq D \text { implies } B^{\downarrow \uparrow} \subseteq D^{\downarrow \uparrow} \\
\text { (idempotency) } & B^{\downarrow \uparrow}=B^{\downarrow \uparrow \downarrow \uparrow}
\end{array}
$$

for all $B, D \subseteq Y$ (analogously for the composition ${ }^{\uparrow} \downarrow$ ).

Sets of attributes satisfying $B=B^{\downarrow \uparrow}$ are called closed sets and they are exactly the intents of formal concepts.

\section{Close-by-One}

In the context of FCA, the foundation of LCM is $\mathrm{CbO} 5^{5}$ Therefore, we firstly turn our attention to $\mathrm{CbO}$.

We start the description of $\mathrm{CbO}$ with a naïve algorithm for generating all closed sets. The naïve algorithm traverses the space of all subsets of $Y$, each subset is checked for closedness and is outputted. This approach is quite inefficient as the number of closed subsets is typically significantly smaller than the number of all subsets.

The algorithm is given by a recursive procedure GenerateFrom, which accepts two attributes:

- $B$ - the set of attributes, from which new sets will be generated.

- $y$ - the auxiliary argument to remember the highest attribute in $B$.

The procedure first checks the input set $B$ for closedness and prints it if it is closed (lines 1,2). Then, for each attribute $i$ higher than $y$ :

- a new set is generated by adding the attribute $i$ into the set $B$ (line 4 );

- the procedure recursively calls itself to process the new set (line 5).

The procedure is initially called with an empty set and zero as its arguments. 

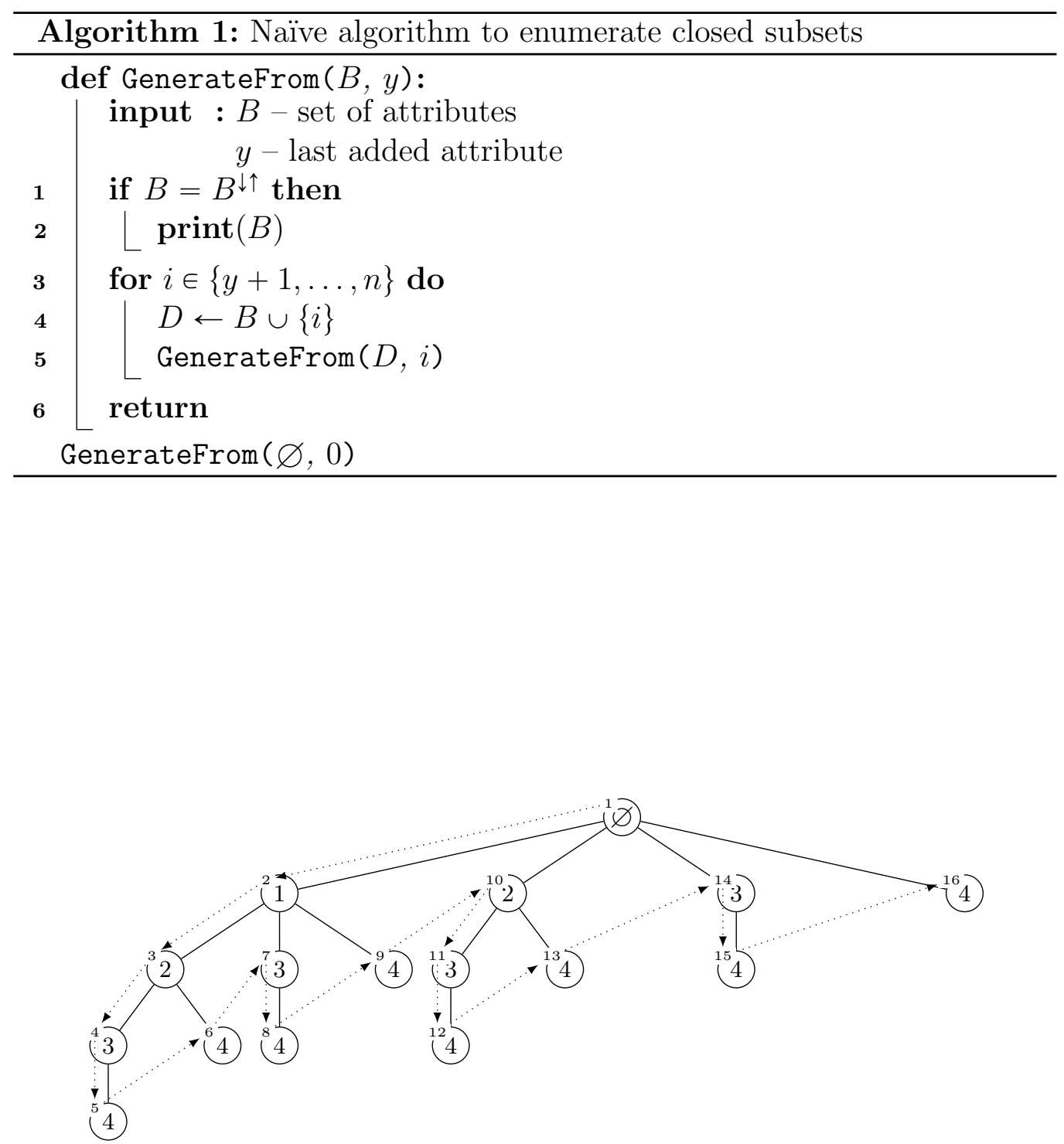

Figure 2: Tree of all subsets of $\{1,2,3,4\}$. Each node represents a unique set containing all elements in the path from the node to the root. The dotted arrows and small numbers represent the order of traversal performed by the algorithm for generating all subsets. 
The naïve algorithm represents a depth-first sweep through the tree of all subsets of $Y$ (see Fig.2) and printing the closed ones.

In the tree of all subsets (Fig.2), each node is a superset of its predecessors. We can use the closure operator ${ }^{\downarrow}$ to skip non-closed sets. In other words, to make jumps in the tree to closed sets only. Instead of simply adding an element to generate a new subset $D \leftarrow B \cup\{i\}$, CbO adds the element and then closes the set

$$
D \leftarrow(B \cup\{i\})^{\downarrow \uparrow}
$$

We need to distinguish the two outcomes of the closure (4). Either

- the closure contains some attributes lower than $i$ which are not included in $B$, i.e. $D_{i} \neq B_{i}$ where $D_{i}=D \cap\{1, \ldots, i-1\}, B_{i}=B \cap\{1, \ldots, i-1\}$;

- or it does not, and we have $D_{i}=B_{i}$.

The jumps with $D_{i} \neq B_{i}$ are not desirable because they land on a closed set which was already processed or will be processed later (depending on the direction of the sweep). CbO does not perform such jumps. The check of the condition $D_{i}=B_{i}$ is called a canonicity test.

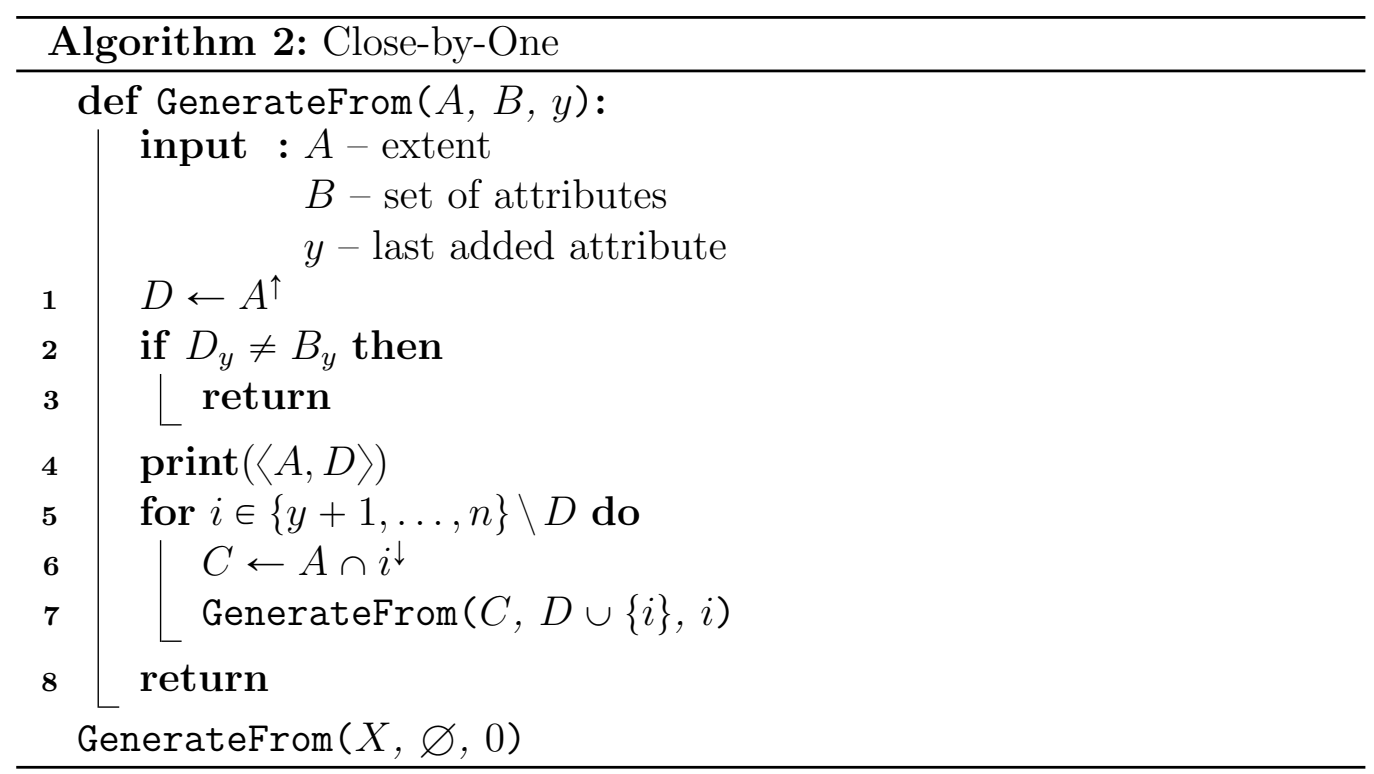

One can see the pseudocode of $\mathrm{CbO}$ in Algorithm 2. In addition, we pass an extent $A$ to the procedure GenerateFrom and the closed set $D\left(=B^{\downarrow \uparrow}\right)$ is

\footnotetext{
${ }^{5}$ It is called a backtracking algorithm with prefix-preserving closure extensions in Uno's papers.
} 
computed as $A^{\uparrow}$ (line 1), which is more efficient. Then the canonicity test is performed. If it fails, the procedure ends (lines 2, 3). Otherwise, we print the concept and continue with the generation of its subtree.

Remark 2. What we show in Algorithm 2 is not the usual pseudocode of $\mathrm{CbO}$ presented in literature. We made some superficial changes to emphasize the link between $\mathrm{CbO}$ and LCM, which will be apparent later. Specifically:

(a) The closure and the canonicity test (lines 1-3) are usually performed before the recursive invocation (line 7 ), not as the first steps of the procedures.

(b) The main loop (line 5) of CbO usually processes the attributes in the ascending order, which corresponds to a left-to-right depth-first sweep through the tree of all subsets (Fig.2). In actual fact, for $\mathrm{CbO}$ there is no reason for a particular order of processing attributes.

\section{Features of LCM}

There are three versions of the LCM algorithm:

LCM1 is $\mathrm{CbO}$ with arraylist representation of data and computing of all extents at once (described in Section 4.2), data preprocessing (described in Section 4.1], and using of diffsets [21] to represent extents for dense data (this is not present in later versions).

LCM2 is LCM1 (without diffsets) with conditional databases (described in Section 4.3

LCM3 is LCM2 which uses a hybrid data structure to represent a context. The data structure uses a combination of FP-trees and bitarrays, called a complete FP-tree (described in Section 6), to handle the most dense attributes. Arraylists are used for the rest, the same way as in the previous versions.

In this paper, we describe all features present in LCM2 and LCM3.

\subsection{Initialization}

To speed the computation up, LCM initializes the input data as follows:

- removes empty rows and columns,

- merges identical rows,

- sorts attributes by cardinality $\left(\left|y^{\downarrow}\right|\right)$ in the descending order, 
- sorts objects by cardinality $\left(\left|x^{\uparrow}\right|\right)$ in the descending order.

In the pseudocode in Algorithm 3 (later in the paper), the initialization is not shown and it is supposed that it is run before the first invocation of the procedure GenerateFrom.

FCA aspect: The attribute sorting is well known to most likely cause a smaller number of computations of closures in CbO-based algorithms [11, 4, 5. This feature is included in publicly available implementations of In-Close4 and $\mathrm{FCbO}$.

The object sorting is a different story. Andrews [4] tested the performance of In-Close2 and concluded that lexicographic order tends to significantly reduce L1 data cache misses. However, the test were made for bitarray representation of contexts.

The reason for object sorting in LCM is probably that a lesser amount of inverses occurs in a computation of a union of rows (shown later (5)), which is consequently easier to sort. Our testing with Uno's implementation of LCM did not show any difference in runtime for unsorted and sorted objects when attributes are sorted. In the implementation of LCM3, the object sorting is not present.

Remark 3. In examples in the present paper, we do not use sorted data, in order to keep the examples small.

\subsection{Ordered arraylists and occurrence deliver}

LCM uses arraylist ${ }^{6}$ as data representation of the rows of the context. It is directly bound to one of the LCM's main features - occurrence deliver:

LCM computes extents $A \cap i^{\downarrow}$ (line 6 in Algorithm 2) all at once using a single traversal through the data. Specifically, it sequentially traverses through all rows $x^{\uparrow}$ of the context and whenever it encounters an attribute $i$, it adds $x$ to an initially empty arraylist - bucket - for $i$ (see Fig.3). As LCM works with conditional datasets (see Section 4.3), attribute extents correspond to extents $A \cap i^{\downarrow}$ (see Algorithm 2, line 6). This is also known as vertical format in DM algorithms; the buckets are also known as tidlists.

LCM generates childs of each node from right to left. That way, it can reuse the memory for extents (buckets). For example, when computing extents in the node $\{2\}$, that is $\{2,3\}^{\downarrow}$ and $\{2,4\}^{\downarrow}$, the algorithm can reuse the memory used by extents $\{3\}^{\downarrow}$ and $\{4\}^{\downarrow}$, because $\{3\}$ and $\{4\}$ (and their subtrees) are already finalized (see Fig. 4).

\footnotetext{
${ }^{6}$ Whenever we write arraylist, we mean ordered arraylists.
} 

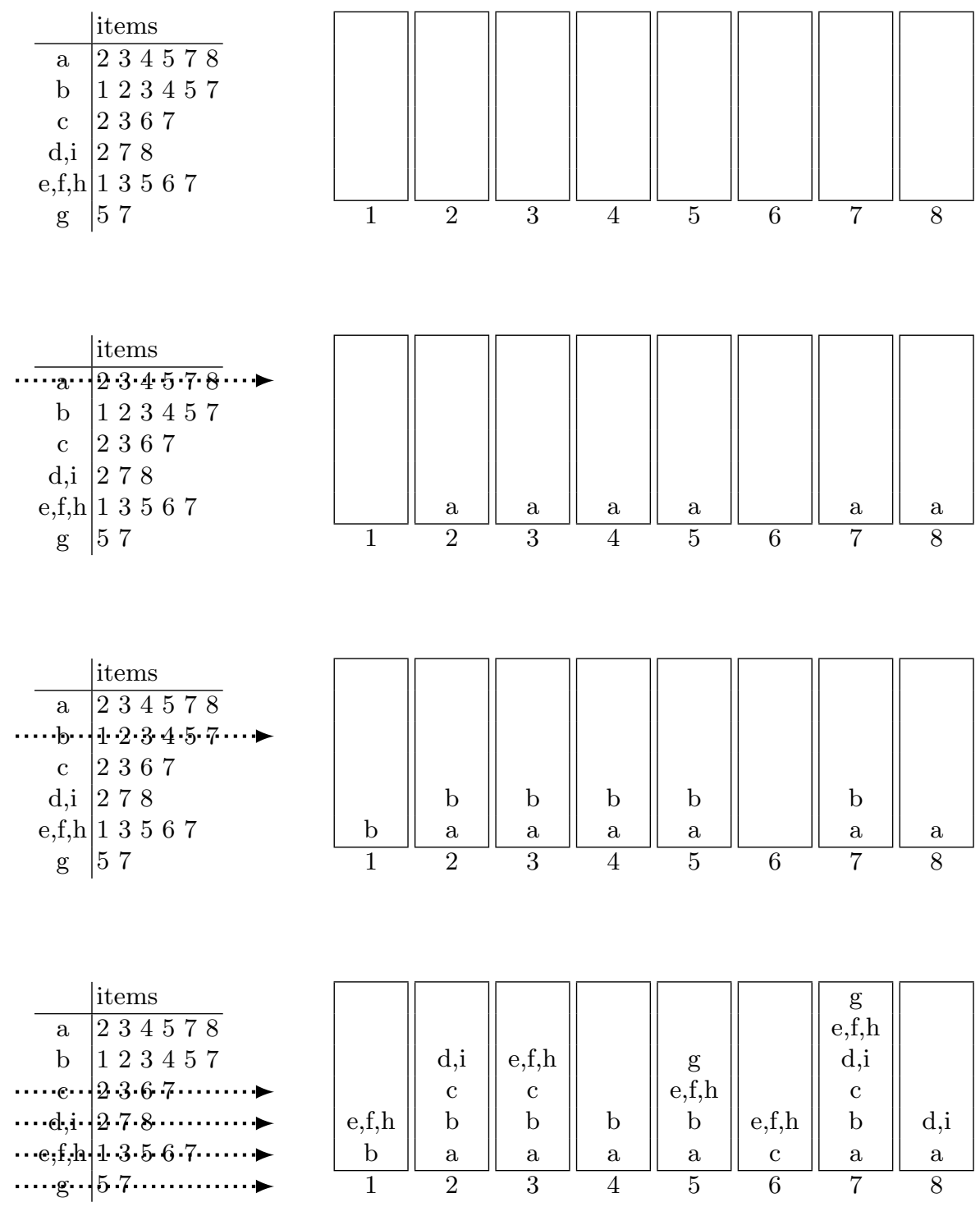

Figure 3: Occurrence deliver in LCM. 


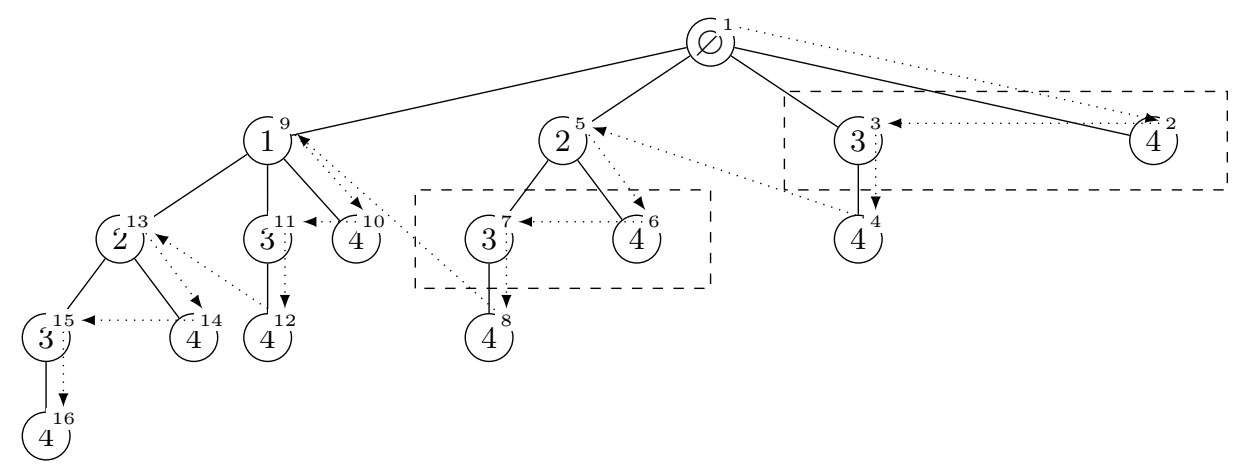

Figure 4: Demonstration of bucket reuse in LCM with right-first sweep.

\begin{tabular}{c|llllll} 
& items & & \\
\hline $\mathrm{a}$ & 2 & 3 & 4 & 5 & 7 & 8 \\
$\mathrm{~b}$ & 1 & 2 & 3 & 4 & 5 & 7 \\
$\mathrm{c}$ & 2 & 3 & 6 & 7 \\
$\mathrm{~d}, \mathrm{i}$ & 2 & 7 & 8 & & \\
$\mathrm{e}, \mathrm{f}, \mathrm{h}$ & 1 & 3 & 5 & 6 & 7 \\
$\mathrm{~g}$ & 5 & 7 & &
\end{tabular}

\begin{tabular}{c|cccccccc} 
& 1 & 2 & 3 & 4 & 5 & 6 & 7 & 8 \\
\hline $\mathrm{a}$ & 0 & 1 & 1 & 1 & 1 & 0 & 1 & 1 \\
$\mathrm{~b}$ & 1 & 1 & 1 & 1 & 1 & 0 & 1 & 0 \\
$\mathrm{c}$ & 0 & 1 & 1 & 0 & 0 & 1 & 1 & 0 \\
$\mathrm{~d}, \mathrm{i}$ & 0 & 1 & 0 & 0 & 0 & 0 & 1 & 1 \\
$\mathrm{e}, \mathrm{f}, \mathrm{h}$ & 1 & 0 & 1 & 0 & 1 & 1 & 1 & 0 \\
$\mathrm{~g}$ & 0 & 0 & 0 & 0 & 1 & 0 & 1 & 0 \\
\hline
\end{tabular}

Figure 5: Data representation for contexts: arraylists (left), bitarrays (right). 
FCA aspect: In FCA, the CbO-based algorithms do not specify data representation used for handling contexts, sets of objects, and sets of attributes. This is mostly considered a matter of specific implementations (see Remark (4). Generally, the data representation issues are almost neglected in literature on FCA. The well-known comparison study [14] of FCA algorithms mentioned the need to study the influence of data structures on practical performances of FCA algorithms but it does not pay attention to that particular issue. The comparison study [12] provided the first steps to an answer for this need.7 The latter paper concludes that binary search trees or linked lists are good choices for large or sparse datasets, while bitarray is an appropriate structure for small or dense datasets. Arraylists did not perform particularly well in any setting. However, this comparison did not assume other features helpful for this data representation, like conditional databases (see Section 4.3) and computation of all required attribute extents in one sweep by occurrence deliver. More importantly, the minimal tested density is $5 \%$, which is still very dense in the context of transactional data.

Remark 4. Available implementations of $\mathrm{FCbO}^{8}$ and In-Close $9^{9}$ utilize bitarrays for rows of contexts, and sets of attributes, and arraylists for sets of objects.

\subsection{Conditional Database and Interior Intersections}

LCM reduces the database for the recursive invocations of GenerateFrom to so-called conditional databases.

Let $\mathcal{K}=\langle X, Y, I\rangle$ be a formal context, $B$ be an intent, and $y$ be the attribute used to build $B$. The conditional database (context) $\mathcal{K}_{B, y}$ w.r.t. $\langle B, y\rangle$ is created from $\mathcal{K}$ as follows:

(a) First, remove objects from $\mathcal{K}$ which are not in the corresponding extent $A=B^{\downarrow}$.

(b) Remove attributes which are full or empty.

(c) Remove attributes lesser than $y \cdot{ }^{10}$

(d) Merge identical objects together.

\footnotetext{
${ }^{7}$ Paper 12 compares bitarrays, sorted linked lists, arraylists, binary search trees, and hash tables.

${ }^{8}$ Available at http://fcalgs. sourceforge.net/

${ }^{9}$ Available at https://sourceforge.net/projects/inclose/.

${ }^{10}$ In the implementation, when the database is already too small (less than 6 objects, and less than 2 attributes), steps (c)-(d) are skipped.
} 
(e) Put back attributes removed in step (c); set each new object created in step (d) by merging objects $x_{1}, \ldots, x_{k} \in X$ to have attributes common to the objects $x_{1}, \ldots, x_{k}$. That is, the merged objects are intersections of the original objects. The part of the context added in this step is called an interior intersection.

For an example, see Fig.6.

Alternatively, we can describe conditional databases with interior intersections as:

- Restricting the context $\mathcal{K}$ to objects in $A$ and attributes in $N$ where

$$
N=\left(\bigcup_{x \in A} x^{\uparrow}\right) \backslash A^{\uparrow} .
$$

This covers the steps (a)-(c).

- Subsequent merging/intersecting of those objects which have the same incidences with attributes in $\{1,2, \ldots, y-1\}$. This covers the steps (d)-(e).

Note, that from the conditional database (with interior intersections) $\mathcal{K}_{B, i}$ all intents in the subtree of $B$ can be extracted. More specifically, if $D$ is an intent of the conditional database and it passes the canonicity test (tested on interior intersections) then $D \cup B$ is an intent of the original context which passes the canonicity test; i.e. is in the subtree of $B$.

In pseudocode in Algorithm 3 (later in the paper), the creation of the conditional databases with interior intersections is represented by the procedure named CreateConditionalDB $(\mathcal{K}, A, N, y)$.

FCA aspect: CbO-based algorithms do not utilize conditional databases. However, we can see partial similarities with features of CbO-based algorithms.

First, all of the algorithms skip attributes in $B$ and work only with part of the formal context given by $B^{\downarrow}$ and $Y \backslash B$. This corresponds to step (a) and the first part of step (b) (full attributes).

Second, the removal of empty attributes in step (b) utilizes basically the same idea as in In-Close4 [6]: if the present extent $A$ and an attribute extent $i^{\downarrow}$ have no common object, we can skip the attribute $i$ in the present subtree. In FCbO and In-Close3, such attribute would be skipped due to pruning (see Section 4.4).

Steps (c)-(e) have no analogy in $\mathrm{CbO}$ algorithms. 


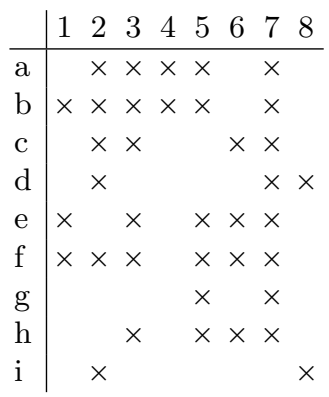

(a) Remove objects not in $\{3,7\}^{\downarrow}$ :

Objects $\mathrm{d}, \mathrm{g}$, and i are removed.

\begin{tabular}{l|llllllll} 
& 1 & 2 & 3 & 4 & 5 & 6 & 7 & 8 \\
\hline $\mathrm{a}$ & & $\times$ & $\times$ & $\times$ & $\times$ & $\times$ \\
$\mathrm{b}$ & $\times$ & $\times$ & $\times$ & $\times$ & $\times$ & $\times$ \\
$\mathrm{c}$ & & $\times$ & $\times$ & & $\times$ & $\times$ & $\times$ \\
$\mathrm{e}$ & $\times$ & $\times$ & $\times$ & $\times$ & $\times$ \\
$\mathrm{f}$ & $\times$ & $\times$ & $\times$ & $\times$ & $\times$ \\
$\mathrm{h}$ & & $\times$ & $\times$ & $\times$ & $\times$
\end{tabular}

(b) Remove empty and full attributes: Attributes 3, 7, and 8 are removed.

\begin{tabular}{l|lllll} 
& 1 & 2 & 4 & 5 & 6 \\
\hline $\mathrm{a}$ & & $\times$ & $\times$ & $\times$ & \\
$\mathrm{b}$ & $\times$ & $\times$ & $\times$ & $\times$ & \\
$\mathrm{c}$ & & $\times$ & & $\times$ \\
$\mathrm{e}$ & $\times$ & & & $\times$ & $\times$ \\
$\mathrm{f}$ & $\times$ & & & $\times$ & $\times$ \\
$\mathrm{h}$ & & & & $\times$ & $\times$
\end{tabular}

(c) Remove attributes lesser than 3: Attributes 1 and 2 are removed.

\begin{tabular}{c|ccc} 
& 4 & 5 & 6 \\
\hline $\mathrm{a}$ & $\times$ & $\times$ & \\
$\mathrm{b}$ & $\times$ & $\times$ & \\
$\mathrm{c}$ & & $\times$ \\
$\mathrm{e}$ & & $\times$ & $\times$ \\
$\mathrm{f}$ & & $\times$ & $\times$ \\
$\mathrm{h}$ & & $\times$ & $\times$
\end{tabular}

(d) Merge identical objects together.

\begin{tabular}{l|rrr} 
& 4 & 5 & 6 \\
\hline $\mathrm{a}, \mathrm{b}$ & $\times$ & $\times$ & \\
$\mathrm{c}$ & & $\times$ \\
$\mathrm{e}, \mathrm{f}, \mathrm{h}$ & & $\times$ & $\times$
\end{tabular}

(e) Add interior intersections.

\begin{tabular}{l|rr|rrrr} 
& 1 & 2 & 4 & 5 & 6 \\
\hline $\mathrm{a}, \mathrm{b}$ & $\times$ & $\times$ & $\times$ & \\
$\mathrm{c}$ & $\times$ & & & $\times$ \\
$\mathrm{e}, \mathrm{f}, \mathrm{h}$ & & & $\times$ & $\times$
\end{tabular}

13

Figure 6: Obtaining contitional context $\mathcal{K}_{B, y}$ for attribute set $B=\{3,7\}$ and attribute $y=3$. 


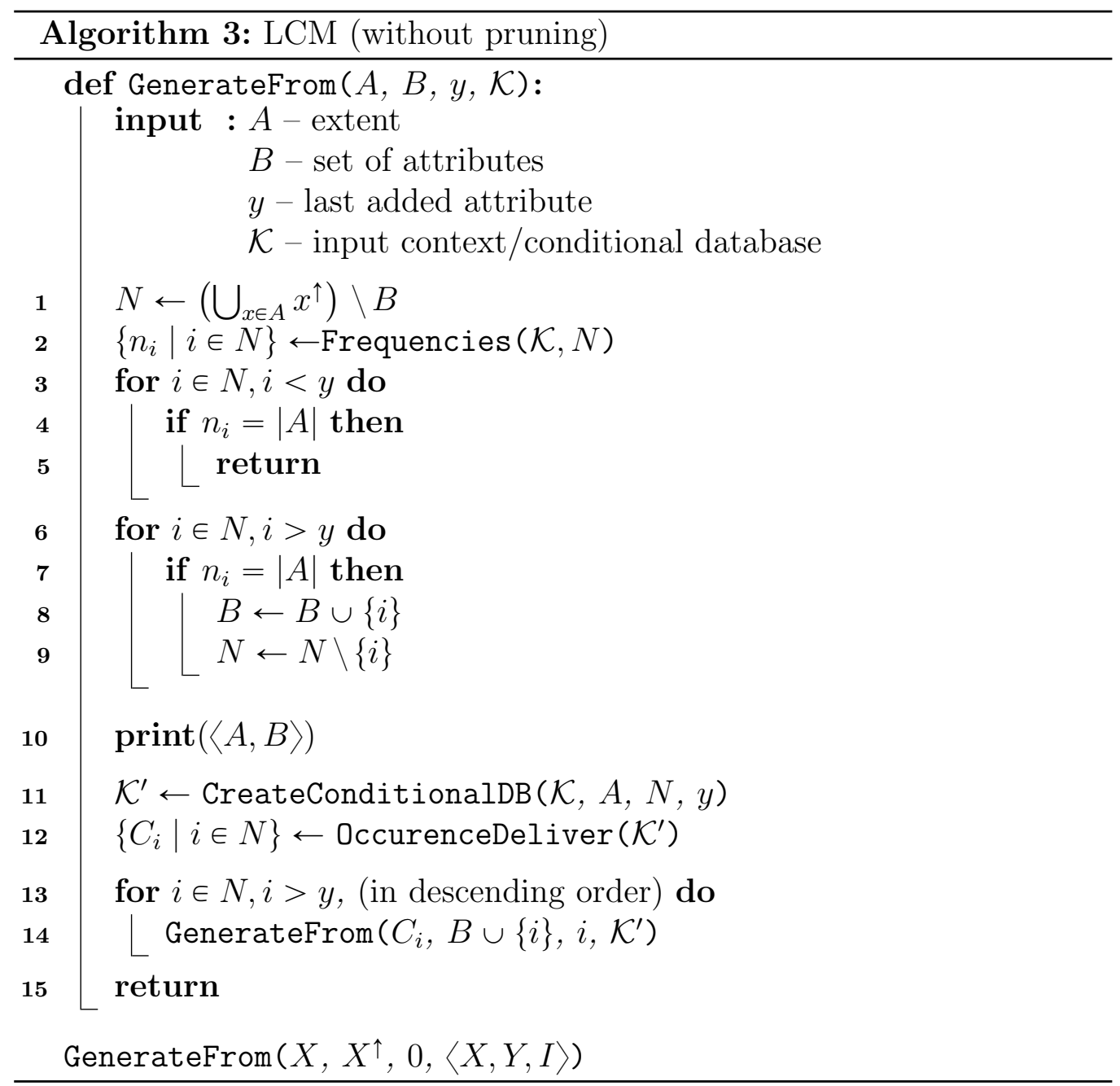

Pseudocode of LCM without pruning

At this moment, we present pseudocode of LCM (Algorithm 3) with above-described features. As in the case for $\mathrm{CbO}$, the algorithm is given by recursive procedure GenerateFrom. The procedure takes four arguments: an extent $A$, a set of attributes $B$, the last attribute $y$ added to $B$, and a (conditional) database $\mathcal{K}$ ). The procedure performs the following steps: 
(line 1) The set $N$ (5) of non-trivial attributes is computed.

(line 2) The frequencies of all attributes in $N$ are computed, this is made by a single traversal through $\mathcal{K}$ similar to the occurence deliver (described in Section 4.2).

(lines $3-5) \quad$ The loop checks whether any attribute in $N$ lesser than $y$ has frequency equal to $|A|$. If so, the attribute causes the canonicity test to fail, therefore we end the procedure.

(lines 6-9) The loop closes $B$ (and updates $N$ ) based on the computed frequencies.

(line 10) As the cannonicity is checked and $B$ is closed, the pair $\langle A, B\rangle$ is printed out.

(line 11) The conditional database $\mathcal{K}_{B, y}$ (described in Sec. 4.3) is created.

(line 12) Attribute extents from $\mathcal{K}_{B, y}$ are computed using occurence deliver (described in Section 4.2).

(lines 13-14) The procedure GenerateFrom is recursively called for attributes in $N$ with the conditional database $\mathcal{K}_{B, y}$ and the corresponding attribute extent.

\subsection{Bonus Feature: Pruning}

The jumps using closures in $\mathrm{CbO}$ significantly reduce the number of visited nodes in comparison with the naïve algorithm. The closure, however, becomes the most time consuming operation in the algorithm. The pruning technique in $\mathrm{LCM}^{11}$ avoids the computation of some closures based on the monotony property: for any set of attributes $B, D \subseteq Y$ satisfying $B \subseteq D$, we have that

$$
j \in(B \cup\{i\})^{\downarrow \uparrow} \quad \text { implies } \quad j \in(D \cup\{i\})^{\downarrow \uparrow} \text {. }
$$

When $i, j \notin D$ and $j<i$, the implication (6) says that if $j$ causes $(B \cup\{i\})^{\downarrow \uparrow}$ to fail the canonicity test, then it also causes $(D \cup\{i\})^{\downarrow \uparrow}$ to fail the canonicity test. That is, if we store that $(B \cup\{i\})^{\downarrow \uparrow}$ failed, we can use it to skip the computation of the closure $(D \cup\{i\})^{\downarrow \uparrow}$ for any $D \supset B$ with $j \notin D$. We demonstrate this in the following example.

Example 1. Consider the following formal-context.

\footnotetext{
${ }^{11}$ Pruning is not described in papers on LCM, however, it is present in the implementation of LCM2.
} 


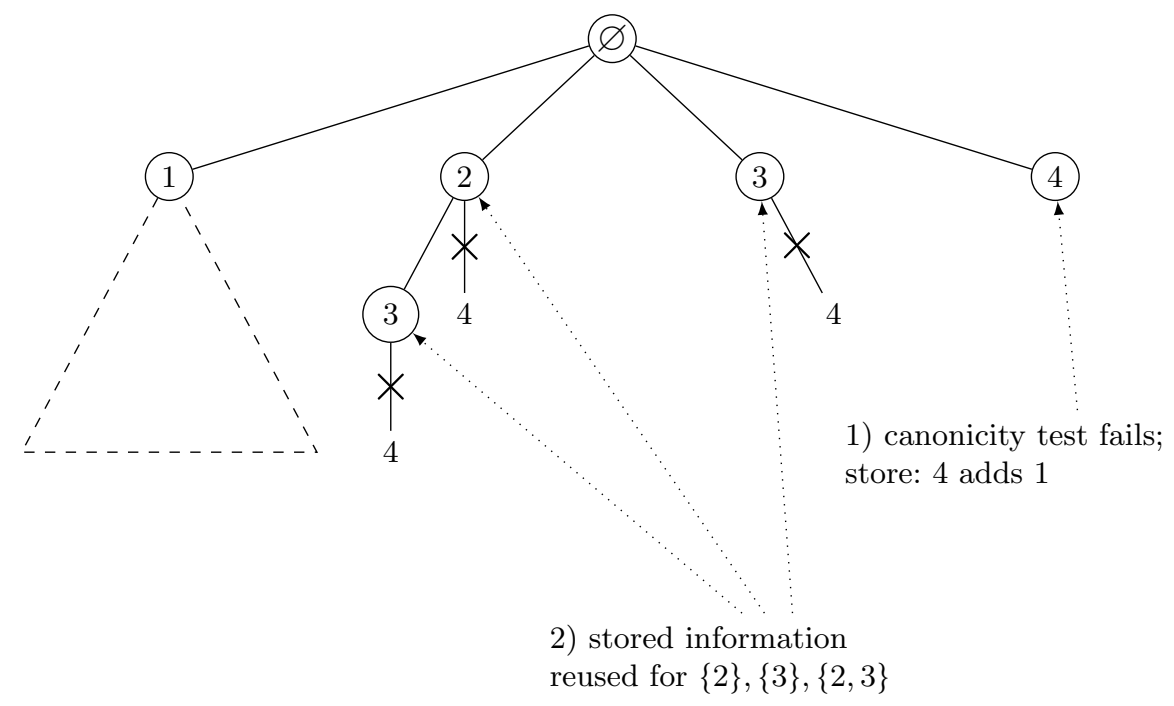

Figure 7: Reuse of failed canonicity test information

$$
\begin{array}{l|llll} 
& 1 & 2 & 3 & 4 \\
\hline \mathrm{a} & \times & \times & \times \\
\mathrm{b} & \times & \times & \times \\
\mathrm{c} & \times & \times & \\
\mathrm{d} & \times & &
\end{array}
$$

Consider the tree of all subsets in Fig.2 (ignoring the left-first sweep order for now). The rightmost branch of the tree represents adding the attribute 4 into an empty set. We can easily see, that

$$
\{4\}^{\downarrow \uparrow}=\{1,4\},
$$

and, therefore, the canonicity test $B_{i}=D_{i}$ fails. In this case, we have $B_{i}=\varnothing_{4}=\varnothing$ while $D_{i}=\{1,4\}_{4}=\{1\}$.

Notice that (7) gives us information about the actual set (an empty set in this case): adding attribute 4 causes that attribute 1 is in the closed set. Due to (6) this holds true for any superset of the actual set. This information is then reused for the supersets. Specifically, for sets $\{2\},\{3\}$, or $\{2,3\}$, adding attribute 4 causes that attribute 1 is present in the closed set and, consequently, causes a failing the canonicity test. Figure 7 shows the described situation.

LCM utilizes the above idea in the following way:

(p0) Whenever the canonicity test fails for $(B \cup\{i\})^{\downarrow \uparrow}$ and $j$ is the smallest attribute in $(B \cup\{i\})^{\downarrow \uparrow} \backslash B$, we store the rule " $i$ adds $j$ ". In the pseudocode (Algorithm 4), this is achieved through the return value of the 
procedure GenerateFrom. The procedure returns the lowest attribute which caused the canonicity to fail (line 6) or 0 if it passed the canonicity test (line 20). The returned value is used to form a pruning rule, which is to be stored (lines 17,18 ).

(p1) At the beginning of GenerateFrom, i.e. when descending to a subtree, all rules having the last added attribute (argument $y$ ) on the right side are removed from the stored rules. In the pseudocode, this is done by a subroutine called RemoveRulesByRightSide (line 3).

(p2) At the end of GenerateFrom, i.e. when backtracking from the current subtree, all rules from this call are removed. In the pseudocode, this is done by a subroutine called RemoveAllRulesAddedThisCall (line 19).

(p3) Before computing a closure $(D \cup\{i\})^{\downarrow \uparrow}$ in a subtree of $B$, we check the stored rules to find whether adding $i$ does not add any attribute which causes the canonicity test to fail. Due to how the rules are handled in the previous items, (p0)-(p2), it is sufficient to check whether there is any rule having $i$ on the left side. In the pseudocode, this is done by a subroutine called CheckRulesByLeftSide (line 15).

FCA aspect:. Similar pruning techniques are also present in FCbO and InClose3 and higher:

- FCbO, In-Close3: stores rules of the form " $i$ gives set $A$ ".

- In-Close4: stores rules of the form " $i$ gives an empty extent".

- In-Close5: stores rules of the form " $i$ adds an attribute which makes the canonicity test fail" and rules of In-Close4.

All the FCA algorithms utilize only steps (p0), (p2), and (p3); none of them performs (p1).

LCM's pruning is weaker than the pruning in FCbO and In-Close3, yet is stronger than the pruning in In-Close 4 and In-Close $5 .{ }^{12}$,

$$
\text { In-Close } 4 \text { In-Close } 5<\text { LCM }<\text { FCbO }=\text { In-Close3. }
$$

\footnotetext{
${ }^{12}$ In the conference paper, we incorrectly claimed that LCM's pruning is incomparable with the pruning in In-Close5.
} 


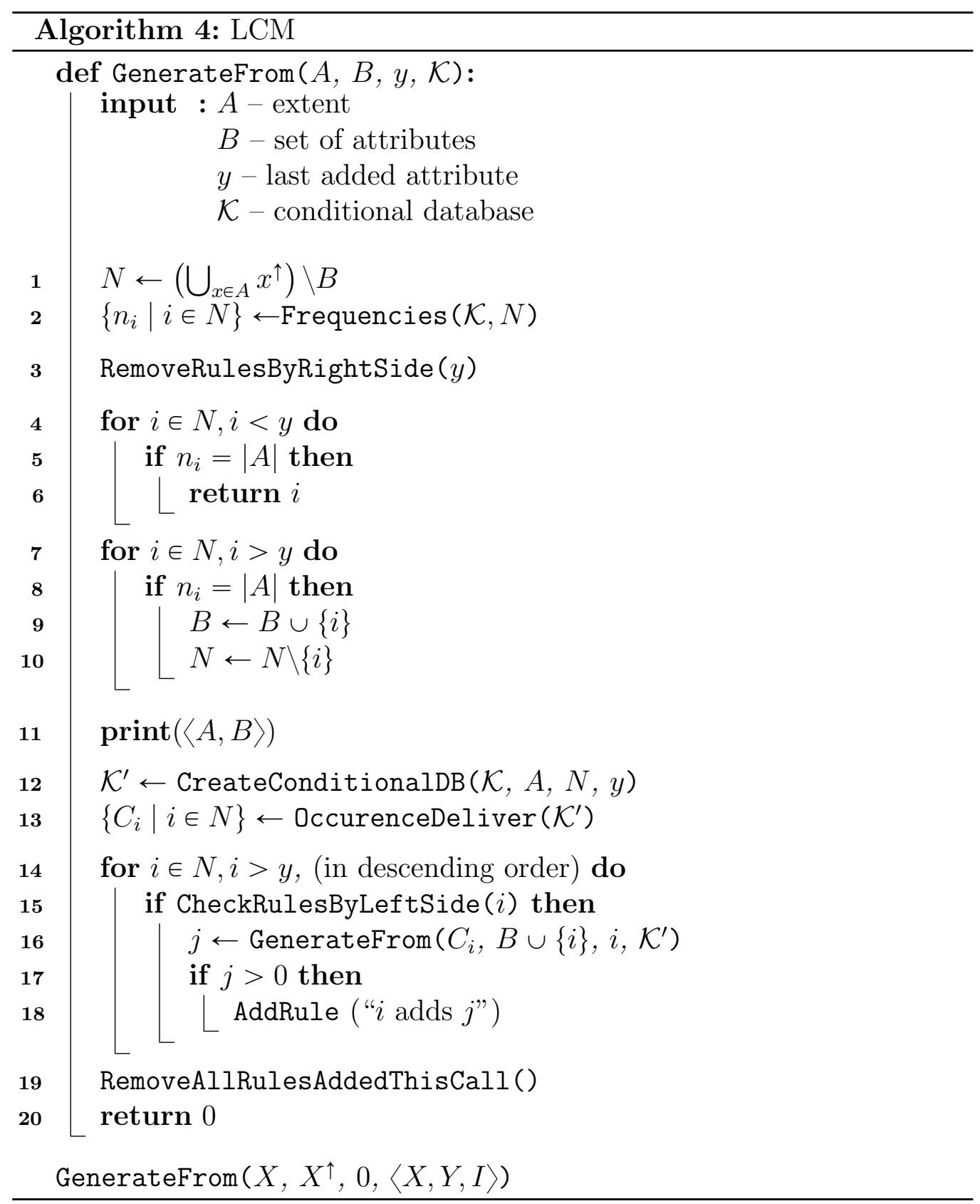




\section{Frequency Counting}

In the previous sections, we do not take into account the frequency of itemsets, as in FCA, the frequency is not usually assumed. However, the implementations of $\mathrm{FCbO}$ and In-Close 4 allow us to pass minimum support as a parameter, and then enumerate only frequent intents.

The CbO-based algorithms utilize a simple apriori principle: if a set is infrequent then all its supersets are infrequent. That directly translates into the tree-like computation of the algorithms - if a node represents an infrequent set, then its subtree does not contain any frequent set.

In LCM, we make the following modification of the features described in Section 4

Data representation. - each arraylist is accompanied with a weight, i.e. number of objects it corresponds to.

Initialization. - additionally, infrequent attributes are removed and the weights of rows are set to reflect the number of merged rows. The weight of unique rows is set to 1 .

Conditional databases. - in the step (b), infrequent attributes are removed as well; and in the step (d), the weights of rows are updated.

\section{FP-trees in LCM3}

Here, we provide a tutorial-like description of the complete FP-trees with inner intersections used in the hybrid data structure of LCM3.

Throughout this section, we assume that the input data does not contain empty rows - they were removed by the initialization procedure (Section 4.1).

\subsection{Simple FP-tree}

An FP-tree [9] (also called a prefix tree) is a compressed representation of the input data. It is constructed by reading the data set one row at a time and mapping each of them onto a path in the FP-tree. The path contains one node for each attribute of the corresponding row, in descending order w.r.t. cardinality of the attributes. Multiple transactions can share part of their paths. Besides their attributes, the nodes contain a number of rows which share the node. For practicality, nodes corresponding to the same attributes are linked to lists.

It is not necessary to use the descending order, but it tends to make the FP-tree more condense. Note that the attributes are sorted by their cardinality in ascending order in the pre-processing step (Section 4.1) and 


\begin{tabular}{c|l} 
object & items \\
\hline 1 & $\mathbf{d}, \mathbf{e}$ \\
2 & $\mathbf{b , c}, \mathbf{d}$ \\
3 & $\mathbf{a}, \mathbf{b}, \mathbf{c}, \mathbf{e}$ \\
4 & $\mathbf{a}, \mathbf{b}, \mathbf{e}$ \\
5 & $\mathbf{c , d}, \mathbf{e}$ \\
6 & $\mathbf{b}, \mathbf{c}, \mathbf{d}, \mathbf{e}$ \\
7 & $\mathbf{e}$ \\
8 & $\mathbf{c , d}, \mathbf{e}$ \\
9 & $\mathbf{b}, \mathbf{d}, \mathbf{e}$ \\
10 & $\mathbf{a}, \mathbf{c}, \mathbf{d}$
\end{tabular}

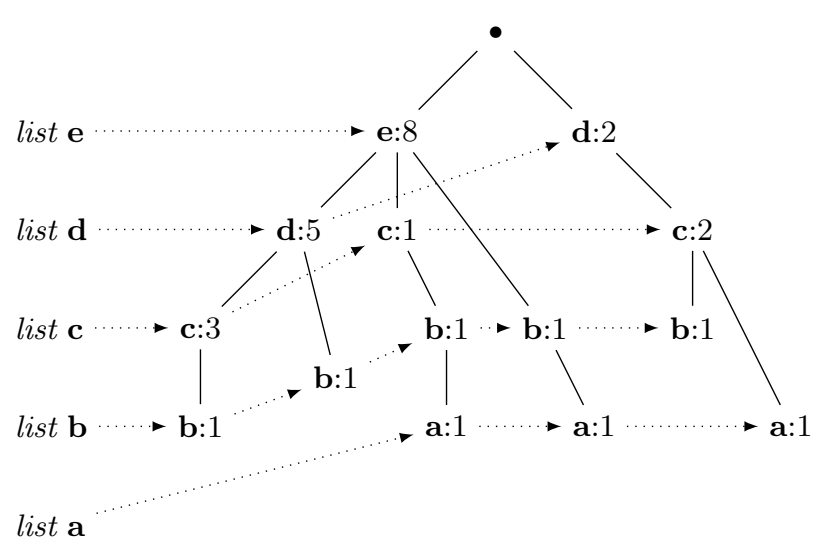

Figure 8: Data (left) and its FP-tree representation (right).

then processed from right to left. This way, they are indeed processed in the descending order.

The following examples depict the construction of an FP-tree and extracting a conditional dataset from it.

Example 2 (Initial construction of an FP-tree). Consider the data depicted in Fig.8. The attributes, sorted by their cardinality, follow the alphabetical order:

$$
\text { (smallest) } \mathbf{a}<\mathbf{b}<\mathbf{c}<\mathbf{d}<\mathbf{e} \text { (largest). }
$$

Therefore, for instance, the first row will be added into the FP-tree as path (root)-(e)-(d). Figure 9 shows the FP-tree after adding the first, second, and third row. Figure 8 (right) shows the FP-tree after adding all the rows.

Example 3 (Extraction of conditional FP-tree). Let us construct a conditional FP-tree for attribute a from the FP-tree in Fig.8 as follows:

(a) Take only the paths which contain the attribute $\mathbf{a}$.

(b) Update numbers to match the numbers of $\mathbf{a}$.

(c) Remove nodes a (and their successors, which is trivial in this case).

(d) Remove infrequent items, if frequency is considered. In Fig.10, 'list d' contains only one node with count 1 . That means that only one row contains $\{\mathbf{a}, \mathbf{d}\}$. If this is seen as infrequent, we can remove the d's node. 

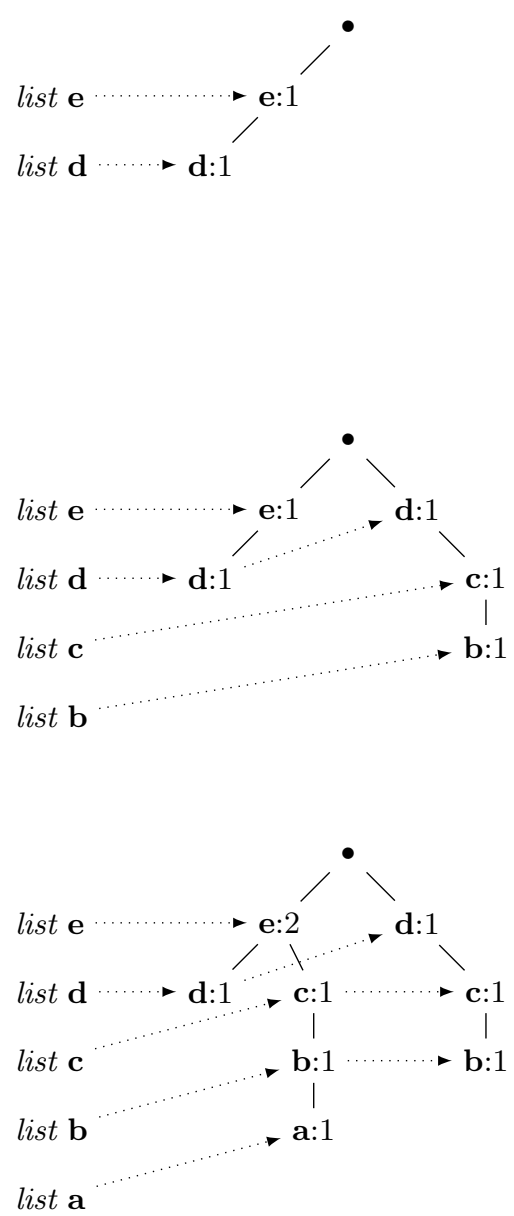

Figure 9: First three steps of the construction of the FP-tree for data from Fig.88. 


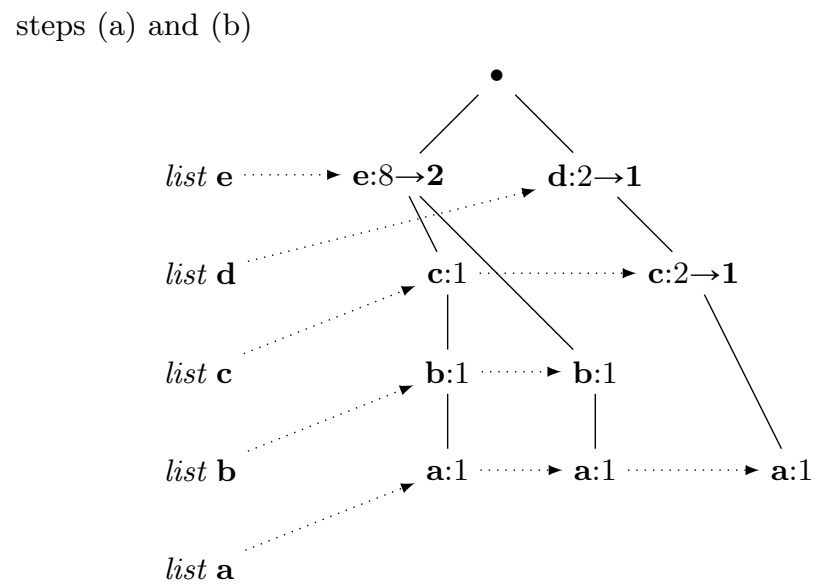

steps (c) and (d)

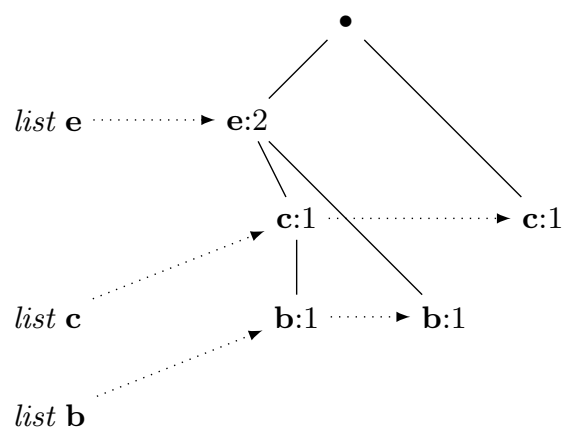

Figure 10: Construction of the conditional FP-tree for attribute a from the FP-tree in Fig.8. 


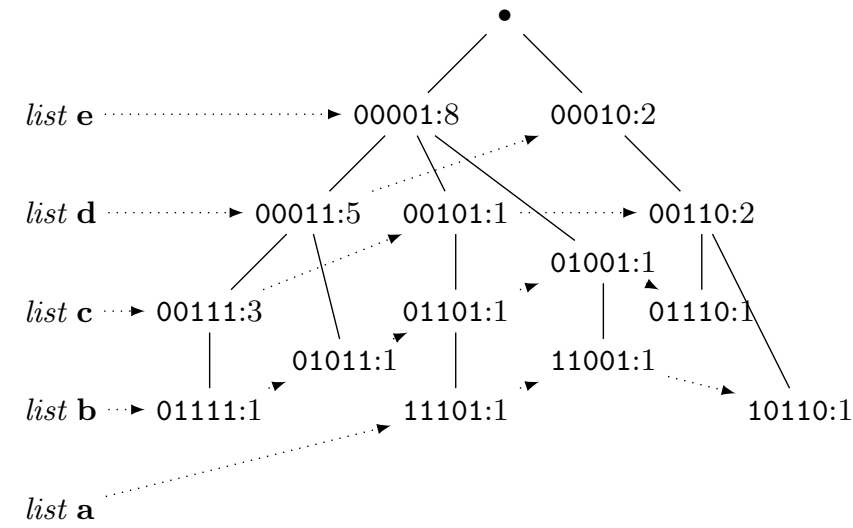

Figure 11: Complete FP-tree corresponding to the FP-tree in Fig.8.

\subsection{Complete FP-trees and inner intersections}

Uno et al. use a so-called complete FP-tree, where each node contains a set of attributes on the path from the node up to the root, stored as a bitarray. See Fig.11 for an example.

Note that in the complete FP-tree, each node has a unique bitarray, which we can use as an identifier. Furthermore, for each non-root node, we have complete information about the related set. We do need to follow the path to the root to find the set. Therefore, we can omit the tree structure, and store just the lists (Fig.12, ignore the rightmost column for now).

The lists play the role of buckets for the occurrence deliver (described in Section 4.2). For example, the 'list c' in Fig. 12 says that the bucket contains $6=3+2+1$ objects:

- three of them have the attributes $\mathbf{c}, \mathbf{d}, \mathbf{e}$;

- two of them have the attributes $\mathbf{c}, \mathbf{d}$ but not $\mathbf{e}$, and

- one has the attributes $\mathbf{c}, \mathbf{e}$ but not $\mathbf{d}$.

We have no information about the presence or absence of attributes $\mathbf{a}$ and $\mathbf{b}$. We will see later that we actually do not need it here, as we will have sufficient information in the inner intersection.

While an FP-tree, or complete FP-tree, is sufficient for mining frequent itemsets, we need additional information for closed sets. Specifically, inner intersections (described in Section 4.3). For that reason, we add to each node an intersection (stored as a bitarray) of all rows which it represents (see Fig.12). 


\begin{tabular}{ll|l} 
& $11101: 1$ & 11101 \\
list $\mathbf{a}$ & $11001: 1$ & 11001 \\
& $10110: 1$ & 10110 \\
\hline & $01110: 1$ & 01110 \\
& $01111: 1$ & 01111 \\
list $\mathbf{b}$ & $01011: 1$ & 01011 \\
& $01101: 1$ & 11101 \\
& $01001: 1$ & 11001 \\
\hline \multirow{2}{*}{ list $\mathbf{c}$} & $00111: 3$ & 00111 \\
& $00110: 2$ & 00110 \\
& $00101: 1$ & 11101 \\
\hline \multirow{2}{*}{ list $\mathbf{d}$} & $00011: 5$ & 00011 \\
& $00010: 2$ & 00110 \\
\hline list $\mathbf{e}$ & $00001: 8$ & 00001
\end{tabular}

Figure 12: Complete FP-tree with inner products.

Example 4. In our running example, the node 00111 represents three rows - namely, 5,6, and 8. Their intersection is $\{\mathbf{c}, \mathbf{d}, \mathbf{e}\}$, therefore, the node has the inner intersection 00111.

\subsection{Construction of the complete FP-tree with inner products}

The construction of the complete FP-tree with inner products has two steps. In the first step (initial step), we put the input data into the corresponding lists. In the second step (extension step), we extend the items in the list to a path to the root.

Initial step: We traverse through the rows of the input data. For each row, we find the list which corresponds to its lowest attribute (with respect to the cardinality order). If there already is a node with the same set in the list, we just increase its counter by on ${ }^{13}$. Otherwise, we make a new node for the set; its counter is set to 1 and its inner product is the same as the row (an intersection of one set is the same set). The node is put in the list.

Example 5. The fifth row in the running example, specifically $\{\mathbf{c}, \mathbf{d}, \mathbf{e}\}$ should be put to 'list c'. We create a new node with set (bitarray) 00111, its counter being set to 1 , and its inner intersection is again 00111. Later, we process the eighth row - also $\{\mathbf{c}, \mathbf{d}, \mathbf{e}\}$. As we already have a node with the set 00111 in 'list c', we just increase its counter by one.

Figure 13 shows an "unfinished" FP-tree after the initial step.

\footnotetext{
${ }^{13}$ or its weight
} 


\begin{tabular}{c|l} 
object & items \\
\hline 1 & $\mathbf{d}, \mathbf{e}$ \\
2 & $\mathbf{b , c}, \mathbf{d}$ \\
3 & $\mathbf{a}, \mathbf{b}, \mathbf{c}, \mathbf{e}$ \\
4 & $\mathbf{a}, \mathbf{b}, \mathbf{e}$ \\
5 & $\mathbf{c , d}, \mathbf{e}$ \\
6 & $\mathbf{b , c}, \mathbf{d}, \mathbf{e}$ \\
7 & $\mathbf{e}$ \\
8 & $\mathbf{c , d}, \mathbf{e}$ \\
9 & $\mathbf{b , d}, \mathbf{e}$ \\
10 & $\mathbf{a}, \mathbf{c}, \mathbf{d}$
\end{tabular}

\begin{tabular}{ll|ll} 
& $11101: 1$ & 11101 & 3 \\
list a & $11001: 1$ & 11001 & 4 \\
& $10110: 1$ & 10110 & 10 \\
\hline & $01110: 1$ & 01110 & 2 \\
list $\mathbf{b}$ & $01111: 1$ & 01111 & 6 \\
& $01011: 1$ & 01011 & 9 \\
\hline list $\mathbf{c}$ & $00111: 2$ & 00111 & 5,8 \\
\hline list $\mathbf{d}$ & $00011: 1$ & 00011 & 1 \\
\hline list $\mathbf{e}$ & $00001: 1$ & 00001 & 7
\end{tabular}

Figure 13: "Unfinished" complete FP-tree with inner intersections after the initial step; the numbers on the right side are the numbers of the row in the original data. They are not part of the FP-tree, they are there just for ease of understanding.

Extension Step: For each list in the descending order (with respect to the cardinality order), do the following: For each node $\langle$ set $A, k, I I\rangle$, where $A$ is a set, $k$ is a counter, and $I I$ is an inner intersection, in the list, make a new set (bitarray) $A^{\prime}$ removing the lowest attribute from the set stored in the node. If the new set $A^{\prime}$ is non-empty, take the list which corresponds to its lowest new attribute. Then, if there is a node with the set $A^{\prime}$ in the list, increase its counter by $k$ and intersect its inner intersection with $I I$. Otherwise, make a node $\left\langle A^{\prime}, k, I I\right\rangle$ and put it in the list.

Example 6. Assuming the result of the initial step in Fig. 13, we take sets in the nodes in 'list a' and remove their lowest attribute (being attribute a). We obtain 01101, 01001, and 00110. The former two belong to 'list b'. There are no nodes with these sets in the list, therefore we create new nodes for them and copy their counters and inner intersection from the original nodes. The last one belongs to 'list c'. Again, there is no node with the set 00110. Therefore we put a new node into it. The intermediate result after extending all nodes in 'list $\mathbf{a}$ ' is shown in Fig. 14(left).

Similarly, we continue with nodes in 'list b' (including the new nodes). When we process the node with set 01110 , we remove its lowest attribute and obtain 00110. We see that it belongs to 'list c' and there is already a node with this set. Therefore, we just increase its counter and intersect its inner intersections with the values from the original node. The intermediate result after extending all nodes in 'list b' is shown in Fig.14(right).

When this step is finished for all the lists, we obtain the complete FP-tree shown in Fig. 12 . 


\begin{tabular}{ll|l} 
& $11101: 1$ & 11101 \\
list $\mathbf{a}$ & $11001: 1$ & 11001 \\
& $10110: 1$ & 10110 \\
\hline & $01110: 1$ & 01110 \\
& $01111: 1$ & 01111 \\
list $\mathbf{b}$ & $01011: 1$ & 01011 \\
& $01101: 1$ & 11101 \\
& $01001: 1$ & 11001 \\
\hline \multirow{2}{*}{ list $\mathbf{c}$} & $00111: 2$ & 00111 \\
& $00110: 1$ & 10110 \\
\hline list $\mathbf{d}$ & $00011: 1$ & 00011 \\
\hline list $\mathbf{e}$ & $00001: 1$ & 00001
\end{tabular}

\begin{tabular}{ll|l} 
& $11101: 1$ & 11101 \\
list $\mathbf{a}$ & $11001: 1$ & 11001 \\
& $10110: 1$ & 10110 \\
\hline & $01110: 1$ & 01110 \\
& $01111: 1$ & 01111 \\
list $\mathbf{b}$ & $01011: 1$ & 01011 \\
& $01101: 1$ & 11101 \\
& $01001: 1$ & 11001 \\
\hline \multirow{3}{*}{ list $\mathbf{c}$} & $00111: 3$ & 00111 \\
& $00110: 2$ & 00110 \\
& $00101: 1$ & 11101 \\
\hline list $\mathbf{d}$ & $00011: 2$ & 00011 \\
\hline list $\mathbf{e}$ & $00001: 2$ & 00001
\end{tabular}

Figure 14: Intermediate result of the construction of the complete FP-tree with inner intersections from Example 6 after extending all nodes in 'list a' (left) and 'list b' (right).

\begin{tabular}{ll|l} 
list $\mathbf{b}$ & $01101: 1$ & 11101 \\
& $01001: 1$ & 11001 \\
\hline list $\mathbf{c}$ & $00110: 1$ & 10110 \\
& $00101: 1$ & 11101 \\
\hline list $\mathbf{d}$ & $00010: 1$ & 10110 \\
\hline list $\mathbf{e}$ & $00001: 2$ & 11001
\end{tabular}

Figure 15: Conditional complete FP-tree for attribute $\mathbf{a}$ with inner intersections extracted from the FP-tree from Fig.12

\subsection{Conditional database}

We already said that the lists of a complete FP-tree serve as the buckets for occurrence deliver. Now, we show that we can easily use them for the construction of the conditional database.

To construct the conditional database for an attribute, simply take its list and perform the extension step of the construction of the FP-tree, and then remove (or just ignore) the list and the lists of lower attributes. If a minimal support is required, we can also remove infrequent lists.

Example 7. In our running example, we can extract a conditional database for attribute a by taking the 'list a', performing the extension step, and removing the 'list a'. The result is shown in Fig. 15. Note, that if we remove the infrequent item $\mathbf{d}$, we receive the same conditional database as in the simple FP-tree in Example 3. 


\begin{tabular}{ll|l} 
& $11101: 1$ & 11101 \\
list $\mathbf{a}$ & $11001: 1$ & 11001 \\
& $10110: 1$ & 10110 \\
\hline & $01110: 1$ & 01110 \\
& $01111: 1$ & 01111 \\
list $\mathbf{b}$ & $01011: 1$ & 01011 \\
& $01101: 1$ & 11101 \\
& $01001: 1$ & 11001 \\
\hline \multirow{2}{*}{ list $\mathbf{c}$} & $00111: 3$ & 00111 \\
& $00110: 2$ & 00110 \\
& $00101: 1$ & 11101 \\
\hline \multirow{2}{*}{ list $\mathbf{d}$} & $00011: 5$ & 00011 \\
& $00010: 2$ & 00110 \\
\hline list $\mathbf{e}$ & $00001: 5$ & 00011
\end{tabular}

\begin{tabular}{ll|l} 
& $11101: 1$ & 11101 \\
list $\mathbf{a}$ & $11001: 1$ & 11001 \\
& $10110: 1$ & 10110 \\
\hline & $01110: 1$ & 01110 \\
& $01111: 1$ & 01111 \\
list $\mathbf{b}$ & $01011: 1$ & 01011 \\
& $01101: 1$ & 11101 \\
& $01001: 1$ & 11001 \\
\hline \multirow{2}{*}{ list $\mathbf{c}$} & $00111: 3$ & 00111 \\
& $00110: 2$ & 00110 \\
& $00101: 1$ & 11101 \\
\hline \multirow{2}{*}{ list $\mathbf{d}$} & $00011: 3$ & 00111 \\
& $00010: 2$ & 00110 \\
\hline list $\mathbf{e}$ & $00001: 4$ & 00101
\end{tabular}

Figure 16: Complete FP-tree with inner intersections where (left): we removed 'list $\mathbf{e}$ ' and used its space for the conditional FP-tree of attribute $\mathbf{d}$; (right): we removed 'list $\mathbf{d}$ ' and 'list $\mathbf{e}$ ' and used their space for the conditional FP-tree of attribute $\mathbf{c}$.

\subsection{Reuse of the lists/buckets}

The occurrence deliver (in Section 4.2) reused buckets when it used the right to left sweep through the search space. A similar principle can be used with an FP-tree. Indeed, whenever the algorithm finishes processing a conditional FP-tree, the space allocated for it can be then used by the next conditional FP-tree. This is better demonstrated in the next example.

Example 8. The conditional FP-tree for $\mathbf{e}$ is trivial: it is an empty tree ${ }^{14}$ When we finish processing the attribute $\mathbf{e}$, we can remove its list and use that space for the conditional FP-tree of attribute $\mathbf{d}$. Note that this conditional FP-tree has only the 'list e'. Figure 16 (left) shows the situation where we removed 'list $\mathbf{e}$ ' and used its space for the conditional FP-tree of attribute $\mathbf{d}$.

Analogously, when we finish processing the attribute $\mathbf{d}$, we can delete the 'list d' and 'list e' and use their space for the conditional FP-tree of attribute c. This is shown in Figure 16 (right).

\subsection{Extraction of intents}

For each list in a (conditional) complete FP-tree, the intersection of inner intersections of its nodes is an intent. LCM can easily compute the intersections and print them out whenever they satisfy the canonicity test.

\footnotetext{
${ }^{14}$ This holds generally true; the highest attribute has empty conditional FP-tree.
} 


\section{Conclusions}

We analyzed LCM from the point of view of FCA and concluded that it is a CbO-based algorithm with additional features directed towards processing sparse data. We also compared the additional features with those of $\mathrm{FCbO}$ and InClose2+ known in the FCA community.

Future research: We see two main directions for our upcoming research:

- The investigation of other algorithms for closed frequent itemset mining and putting them into context with FCA algorithms.

- Experimental evaluation of the incorporation of LCM's features in CbO-based algorithms; this could lead to fast implementations of the algorithms.

Acknowledgment: The authors acknowledge support by the grants:

- IGA 2020 of Palacký University Olomouc, No.IGA_PrF_2020_019,

- JG 2019 of Palacký University Olomouc, No. JG_2019_008.

\section{References}

[1] Rakesh Agrawal, Heikki Mannila, Ramakrishnan Srikant, Hannu Toivonen, A Inkeri Verkamo, et al. Fast discovery of association rules. Advances in knowledge discovery and data mining, 12(1):307-328, 1996.

[2] Gabriela Alexe, Sorin Alexe, Tibérius O Bonates, and Alexander Kogan. Logical analysis of data-the vision of Peter L. Hammer. Annals of Mathematics and Artificial Intelligence, 49(1-4):265-312, 2007.

[3] Gabriela Alexe and Peter L. Hammer. Spanned patterns for the logical analysis of data. Discrete Applied Mathematics, 154(7):1039-1049, 2006.

[4] Simon Andrews. In-Close2, a high performance formal concept miner. In Proceedings of the 19th International Conference on Conceptual Structures for Discovering Knowledge, ICCS'11, pages 50-62, Berlin, Heidelberg, 2011. Springer-Verlag.

[5] Simon Andrews. A 'Best-of-Breed' approach for designing a fast algorithm for computing fixpoints of Galois connections. Information Sciences, 295:633-649, 2015. 
[6] Simon Andrews. Making use of empty intersections to improve the performance of CbO-type algorithms. In International Conference on Formal Concept Analysis, pages 56-71. Springer, 2017.

[7] Simon Andrews. A new method for inheriting canonicity test failures in Close-by-One type algorithms. In CLA, volume 2123, pages 255-266, 2018 .

[8] Roberto J. Bayardo Jr. Efficiently mining long patterns from databases. In ACM Sigmod Record, volume 27, pages 85-93. ACM, 1998.

[9] Jiawei Han, Jian Pei, and Yiwen Yin. Mining frequent patterns without candidate generation. ACM sigmod record, 29(2):1-12, 2000.

[10] Radek Janostik, Jan Konecny, and Petr Krajča LCM is Well Implemented CbO: Study of LCM from FCA Point of View In CLA 2020, CEUR Workshop Proceedings, volume 2668, pages 47-58. CEURWS.org, 2020.

[11] Petr Krajca, Jan Outrata, and Vilem Vychodil. Advances in algorithms based on CbO. In CLA, volume 672, pages 325-337, 2010.

[12] Petr Krajca and Vilem Vychodil. Comparison of data structures for computing formal concepts. In International Conference on Modeling Decisions for Artificial Intelligence, pages 114-125. Springer, 2009.

[13] Sergei O. Kuznetsov. A fast algorithm for computing all intersections of objects from an arbitrary semilattice. Nauchno-Tekhnicheskaya Informatsiya Seriya 2-Informatsionnye Protsessy i Sistemy, (1):17-20, 1993.

[14] Sergei O. Kuznetsov and Sergei Obiedkov. Comparing performance of algorithms for generating concept lattices. Journal of Experimental and Theoretical Artificial Intelligence, 14:189-216, 2002.

[15] Heikki Mannila and Hannu Toivonen. Multiple uses of frequent sets and condensed representations. In KDD, volume 96, pages 189-194, 1996.

[16] Jan Outrata and Vilem Vychodil. Fast algorithm for computing fixpoints of Galois connections induced by object-attribute relational data. Information Sciences, 185(1):114-127, 2012.

[17] Takeaki Uno, Tatsuya Asai, Yuzo Uchida, and Hiroki Arimura. LCM: An efficient algorithm for enumerating frequent closed item sets. In FIMI, volume 90. Citeseer, 2003. 
[18] Takeaki Uno, Tatsuya Asai, Yuzo Uchida, and Hiroki Arimura. An efficient algorithm for enumerating closed patterns in transaction databases. In International Conference on Discovery Science, pages 16-31. Springer, 2004.

[19] Takeaki Uno, Masashi Kiyomi, and Hiroki Arimura. LCM ver. 2: Efficient mining algorithms for frequent/closed/maximal itemsets. In FIMI, volume 126, 2004.

[20] Takeaki Uno, Masashi Kiyomi, and Hiroki Arimura. LCM ver. 3: collaboration of array, bitmap and prefix tree for frequent itemset mining. In Proceedings of the 1st international workshop on open source data mining: frequent pattern mining implementations, pages 77-86. ACM, 2005.

[21] Mohammed J. Zaki and Karam Gouda. Fast vertical mining using diffsets. In Proceedings of the ninth ACM SIGKDD international conference on Knowledge discovery and data mining, pages 326-335. ACM, 2003. 